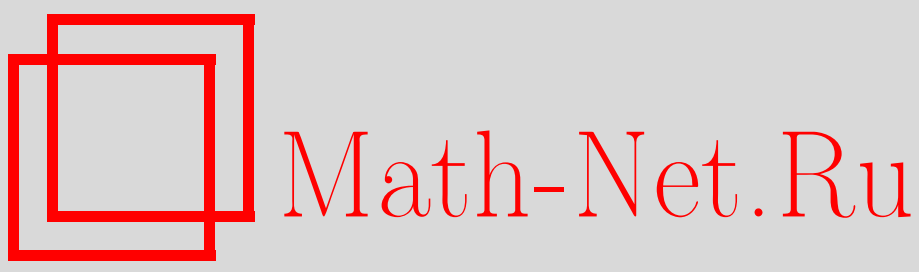

Р. С. Юлмухаметов, Разложение целых функций на произведение двух функций эквивалентного роста, Матем. сб., 1996, том 187, номер 7, 139-160

DOI: https://doi.org/10.4213/sm149

Использование Общероссийского математического портала Math-Net.Ru подразумевает, что вы прочитали и согласны с пользовательским соглашением http://www . mathnet.ru/rus/agreement

Параметры загрузки:

IP : 54.166 .219 .16

26 апреля 2023 г., 14:03:51 
УДК 517.547

\section{Р.С. Юлмухаметов}

\section{Разложение целых функций на произведение двух функций эквивалентного роста}

Работа посвящена проблеме Л. Эйренпрайса о факторизации в алгебре гладких финитных функций относительно операции свертки. В начале 80 -х годов было доказано, что не всякая гладкая финитная функция в $\mathbb{R}^{n}, n \geqslant 2$, может быть представлена как свертка двух гладких финитных функций. Д.Г. Диксоном показано, что гладкая финитная функция одной переменной представляется как свертка двух гладких финитных функций, если нули преобразования Фурье-Лапласа этой функции лежат в некоторой горизонтальной полосе и выполнено условие

$$
\sum_{\left|\lambda_{k}\right| \leqslant r} 1=D r+O(1), \quad r \rightarrow \infty
$$

где $\lambda_{k}$ - нули преобразования Фурье-Лапласа.

В данной работе доказывается, что факторизация возможна при условии: все нули преобразования Фурье-Лапласа лежат в области вида

$$
G_{a}=\{z=x+i y,|y| \leqslant \exp (a \sqrt{\ln (|x|+1)})\} .
$$

Библиографйл: 11 названий.

0. Введение. Пусть $\mathscr{D}\left(\mathbb{R}^{n}\right)$ - пространство бесконечно дифференцируемых функций с компактным носителем в $\mathbb{R}^{n}$. Если $\varphi_{1}, \varphi_{2}-$ функции из $\mathscr{D}\left(\mathbb{R}^{n}\right)$, то их свертка

$$
\left(\varphi_{1} * \varphi_{2}\right)(x)=\int \varphi_{1}(x-t) \varphi_{2}(t) d t
$$

также является элементом $\mathscr{D}\left(\mathbb{R}^{n}\right)$. В работе [1] Л. Эйренпрайс поставил вопрос: можно ли любую функцию из $\mathscr{D}\left(\mathbb{R}^{n}\right)$ представить в виде свертки двух функций из $\mathscr{D}\left(\mathbb{R}^{n}\right)$ ? С помощью преобразования Фурье-Лапласа эту проблему можно сформулировать в терминах целых функций. Пусть $\widehat{\varphi}(\lambda)$ обозначает преобразование Фурье-Лапласа функции $\varphi \in \mathscr{D}\left(\mathbb{R}^{n}\right)$ :

$$
\widehat{\varphi}(\lambda)=\int \varphi(t) \exp \langle i \lambda, t\rangle d t, \quad \lambda \in \mathbb{C}^{n}, \quad\langle\lambda, t\rangle=\sum_{k=1}^{n} \lambda_{k} t_{k},
$$

и

$$
\widehat{\mathscr{D}}\left(\mathbb{R}^{n}\right)=\left\{\widehat{\varphi}(\lambda), \varphi \in \mathscr{D}\left(\mathbb{R}^{n}\right)\right\}
$$

По теореме Пэли-Винера-Шварца $\widehat{\mathscr{D}}\left(\mathbb{R}^{n}\right)$ совпадает с классом целых функций $f$ экспоненциального типа в $\mathbb{C}^{n}$, удовлетворяющих условию

$$
|f(x)|=O\left(|x|^{-N}\right), \quad|x| \rightarrow \infty, \quad x \in \mathbb{R}^{n}, \quad \forall N \in \mathbb{N}
$$


(см. [2]). Кроме того, как известно,

$$
\widehat{\varphi_{1} * \varphi_{2}}=\widehat{\varphi}_{1} \cdot \widehat{\varphi}_{2} \text {. }
$$

Двойственная постановка проблемы Эйренпрайса теперь звучит так: можно ли любую целую функцию экспоненциального типа в $\mathbb{C}^{n}$, удовлетворяющую условию (1), представить в виде произведения двух целых функций, обладающих теми же свойствами?

В работах [3]-[5] получен отрицательный ответ на этот вопрос при $n \geqslant 2$. В $\mathscr{D}(\mathbb{R})$ вопрос остается открытым. В работе [6] Д.Г. Диксон показал, что если нули функции $\widehat{\varphi}$ расположены в некоторой горизонтальной полосе и

$$
\sum_{\left|\lambda_{k}\right| \leqslant r} 1=D r+O(1), \quad r \rightarrow \infty
$$

то функция $\varphi$ представляется в виде свертки двух функций из $\mathscr{D}(\mathbb{R})$. В работе [7] доказана возможность факторизации в более обшей ситуации. Автору в [9] удалось показать, что в теореме Д.Г. Диксона расположение нулей в полосе уже является достаточным условием для факторизации и, тем самым, условие (2) в этом случае излишне. В данной работе мы рассмотрим вопрос о факторизации целых функций с нулями, лежашими в области более широкой, чем полоса. Будет доказана следуюшая, основная для этой работы, теорема.

Теорема. Пусть $f$ - целая функиия, все нули которой лежат в области

$$
G_{a}=\{z=x+i y,|y| \leqslant \exp (a \sqrt{\ln (|x|+1)})\}
$$

где а > 0 - некоторая постоянная. Предположим также, что существует целая функция $F(\lambda), F(0)=1$, делящаяся на функцию $f(\lambda)$ и удовлетворяющая условию

$$
\ln |F(\lambda)| \leqslant H(\lambda), \quad \lambda \in \mathbb{C},
$$

где $H(\lambda)$ - некоторая липшицева функция:

$$
|H(\lambda)-H(z)| \leqslant \sigma|\lambda-z|, \quad \lambda, z \in \mathbb{C} .
$$

Тогда функиия $f$ представляется в виде произведения двух цельх функций $f_{1}$, $f_{2}$ таких, что для любого $p>1$ выполняется соотночение

$$
\begin{aligned}
|\ln | f_{1}(x)|-\ln | f_{2}(x)|| \leqslant \frac{C_{0}}{p} & {[H(x)-\ln |F(x)|] } \\
& +C_{1} \ln ^{+} \frac{1}{\rho(x)}+C_{2} \ln (|x|+1)+C_{3} e^{p}, \quad x \in \mathbb{R},
\end{aligned}
$$

где $\rho(\lambda)$ - расстояние от точки $\lambda$ до множества нулей функиии $f, C_{i}-$ постоянные, не зависящие от $p$. Кроме того, для всех $z=r \exp (i \varphi), \varphi \neq 0$, $\pi$ и $\varepsilon>0$ выполняется соотночение

$$
|\ln | f_{1}(x)|-\ln | f_{2}(x)|| \leqslant C(\varphi, \varepsilon)|\lambda|^{\varepsilon}, \quad|\lambda| \geqslant r(\varphi, \varepsilon) .
$$


Эта теорема в некоторых случаях позволяет решить проблему Л. Эйренпрайса. Например, если $\varphi \in \mathscr{D}(\mathbb{R})$ и все нули функции $\widehat{\varphi}$ лежат в области типа $G_{a}$, то функция $\varphi$ представима в виде свертки двух функций из $\mathscr{D}(\mathbb{R})$. В действительности эта теорема позволяет решить вопрос о факторизации и в других классах функций.

Прежде чем приступить к доказательству основной теоремы, сделаем следующее замечание. Если функция $f$ в условии теоремы является произведением целых функций $u$ и $v$, то каждая из функций $u$ и $v$ удовлетворяет условиям теоремы, и если для каждой из этих функций доказано утверждение теоремы, то утверждение будет доказано и для функции $f$. Например, функцию $f$ представим в виде произведения целых функций $u$ и $v$ так, что все нули функции $u$ имеют четную кратность, а нули функции $v$ все простые. Тогда функция $u$ является квадратом целой функции и утверждение теоремы для нее выполняется тривиальным образом. Исходя из этого мы можем с самого начала считать, что все нули функции $f$ простые. Далее, представим функцию $f$ как произведение многочлена $P$ и целой функции $g$ так, что нули многочлена $P$ в точности совпадают с нулями функции $f$ в круге $\{z:|z| \leqslant 2\}$. Снова утверждение теоремы тривиально вьполняется для многочлена $P$, и мы можем считать, что все нули функции $f$ лежат вне круга радиуса 2.

Дальнейшие выводы из этого замечания требуют более тщательного изложения разбиения множества нулей функции $f$ на части с теми или иными дополнительными свойствами.

Положим

$$
a(x)=\exp (a \sqrt{\ln (x+1)}), \quad x \geqslant 0,
$$

и построим последовательность $x_{n}, n=0,1, \ldots$, следуюшим образом. Возьмем $x_{0}=0$, и $x_{1}$ выберем из условия

$$
a\left(x_{1}\right)=x_{1}-x_{0}+1 .
$$

Если выбраны точки $x_{1}, \ldots, x_{n-1}$, то точку $x_{n}$ найдем из условия

$$
a\left(x_{n}\right)=x_{n}-x_{n-1}+1 .
$$

Рассмотрим квадраты

$$
\begin{aligned}
& K_{n}=\{z=x+i y: 2 n \leqslant x<2(n+1),|y| \leqslant 1\}, \quad n \in \mathbb{Z}, \\
& Q_{n}=\left\{z=x+i y: x_{n-1} \leqslant x<x_{n},|y| \leqslant 1\right\}, \quad n \in \mathbb{N},
\end{aligned}
$$

а также квадраты $Q_{n}^{\prime}$, симметричные квадратам $Q_{n}$ относительно вешественной оси, и квадраты $-Q_{n},-Q_{n}^{\prime}$. Объединение всех этих квадратов содержит область $G_{a}$ и, следовательно, содержит все нули функции $f$. Это объединение само содержится в области

$$
\widetilde{G}_{a}=\{z=x+i y:|y|<A \exp (a \sqrt{\ln (|x|+1)})\},
$$

где $A>0$ - некоторая постоянная. Действительно, объединение всех квадратов содержится в области $\{z=x+i y:|y| \leqslant \widetilde{a}(|x|)\}$, где

$$
\widetilde{a}(x)=x_{n}-x_{n-1}+1, \quad x_{n-1}<x \leqslant x_{n} .
$$


Так как $x_{n} \rightarrow \infty$ при $n \rightarrow \infty$, то

$$
\frac{a\left(x_{n+1}\right)}{a\left(x_{n}\right)}=\exp \left[\frac{a\left(\ln \frac{x_{n+1}+1}{x_{n}+1}\right)}{\sqrt{\ln \left(x_{n}+1\right)}+\sqrt{\ln \left(x_{n+1}+1\right)}}\right] \rightarrow 1 .
$$

Поэтому для некоторой постоянной $A$ имеем

$$
a\left(x_{n+1}\right) \leqslant A a\left(x_{n}\right), \quad n=0,1, \ldots
$$

Таким образом, если $x \in\left[x_{n-1}, x_{n}\right]$, то

$$
\widetilde{a}(x)=a\left(x_{n}\right) \leqslant A a\left(x_{n-1}\right) \leqslant A a(x), \quad n=0,1, \ldots .
$$

Множество $\{|y| \leqslant \widetilde{a}(|x|)\}$ в точности совпадает с объединением всех квадратов $K_{n}, \pm Q_{n}, \pm Q_{n}^{\prime}$. Последняя оценка показывает, что это объединение содержится в области $\widetilde{G}_{a}$ :

$$
\left(\bigcup_{n=-\infty}^{\infty} K_{n}\right) \cup\left(\bigcup_{n=0}^{\infty}\left(Q_{n} \cup Q_{n}^{\prime} \cup\left(-Q_{n}^{\prime}\right) \cup\left(-Q_{n}\right)\right)\right) \subset \widetilde{G}_{a} .
$$

Множество нулей $\Lambda$ функции $f$ разобъем на непересекающиеся части следующим образом. Через $\Lambda_{0}$ обозначим максимальное подмножество $\Lambda$, пересечение которого с каждым из квадратов $K_{n}, \pm Q_{n}, \pm Q_{n}^{\prime}$ состоит из четного числа точек. Через $\Lambda_{1}$ обозначим пересечение $\Lambda \backslash \Lambda_{0}$ с объединением всех квадратов $K_{n}$. Тогда в каждом квадрате $K_{n}$ находится не более одной точки из $\Lambda_{1}$. Через $\Lambda_{2}$ обозначим оставшуюся часть $\Lambda: \Lambda_{2}=\Lambda \backslash\left(\Lambda_{0} \cup \Lambda_{1}\right)$. Таким образом, множество $\Lambda_{2}$ содержится в объединение квадратов $\pm Q_{n}, \pm Q_{n}^{\prime}$, и в каждом из этих квадратов находится не более одной точки из $\Lambda_{2}$. Соответственно этому разбиению множества нулей сама функция $f$ может быть представлена как произведение трех целых функций с множеством нулей $\Lambda_{0}, \Lambda_{1}, \Lambda_{2}$. Каждая из этих трех целых функций удовлетворяет условиям основной теоремы, и мы будем доказывать теорему для каждой из них по отдельности. В первом пункте рассмотрим функцию с множеством нулей типа $\Lambda_{0}$.

1. Воспользуемся теоремой 1 из работы [8].

Теорема А. Пусть мера $\mu$ - неотрицательная борелевская мера на плоскости с компактным носителем в квадрате $K$ и $\mu\{z\}<1$ для любой точки $z$. Если полная масса $\mu(\mathbb{C})$ меры $\mu$ - челое число $N$, то мера $\mu$ может быть представлена как сумма неотрицательных мер $\mu_{k} c$ единичной полной массой. Причем существует система прямоугольников $P_{k} \subset K, k=1,2, \ldots, N$, удовлетворяющая следующим условиям.

Р0. Носитель меры $\mu_{k}$ содержится в прямоугольнике $P_{k}$.

P1. Сторонь прямоугольников $P_{k}$ параллельнь сторонам квадрата $K$. Отношение большей стороньи прямоугольника $P_{k} \kappa$ меньшей стороне не превосходит трех.

P2. Каждая точка плоскости попадает во внутренность не более чем четырех прямоугольников $P_{k}$. 
(В работе [8] теорема доказана для непрерывных мер $\mu$. Здесь условие непрерывности заменено на условие $\mu(\{z\})<1$. Доказательство при такой замене условий не меняется.)

В качестве квадрата $K$ возьмем один из квадратов $K_{n}, \pm Q, \pm Q_{n}^{\prime}$, а в качестве меры $\mu$ возьмем атомарную меру, сосредоточенную в точках $\Lambda_{0} \cap K$ и имеющую в каждой из этих точек массу, равную $1 / 2$. Теорема A, примененная в этой ситуации, означает, что точки множества $\Lambda_{0} \cap K$ можно разбить на пары $\lambda_{k}^{\prime}, \lambda_{k}^{\prime \prime}$ так, что сушествует система прямоугольников $P_{k} \subset K, \lambda_{k}^{\prime}, \lambda_{k}^{\prime \prime} \in P_{k}$, удовлетворяюшая условиям P1, P2. Так как эту процедуру можно проделать в каждом из квадратов $K_{n}, \pm Q_{n}, \pm Q_{n}^{\prime}$, то все множество $\Lambda_{0}$ разбивается на пары $\lambda_{k}^{\prime}, \lambda_{k}^{\prime \prime}$, покрытые прямоугольниками $P_{k}$, удовлетворяющими условиям $\mathrm{P} 1, \mathrm{P} 2$. Множество $\Lambda_{0}$ разобьем на две непересекающиеся части. Через $\Lambda_{0}^{\prime}$ обозначим те точки из $\Lambda_{0}$, которые входят в пары, покрытые прямоугольниками с диаметром, большим, чем 3 , а через $\Lambda_{0}^{\prime \prime}$ - множество остальных точек $\Lambda_{0}$. Целая функция с множеством нулей $\Lambda_{0}$, удовлетворяюшая условиям основной теоремы, разлагается на произведение двух функций с множеством нулей $\Lambda_{0}^{\prime}$ и $\Lambda_{0}^{\prime \prime}$. Каждая из этих целых функций удовлетворяет условиям основной теоремы, и эту теорему будем доказывать для каждой из них по отдельности.

1.1. Здесь рассмотрим вопрос о факторизации целой функции с множеством нулей типа $\Lambda_{0}^{\prime \prime}$.

Теорема 1. Пусть $f$ - целая функция, $\lambda_{k}, k \in \mathbb{N},-$ нули функиии $f$. Предположим, что нули функции $f$ разбиты на пары $\lambda_{k}^{\prime}, \lambda_{k}^{\prime \prime}, k \in \mathbb{N}$, так, что существует система прямоугольников $P_{k}$, обладающая следующими свойствами.

Р0. Точки $\lambda_{k}^{\prime}, \lambda_{k}^{\prime \prime}$ лежат в прямоугольнике $P_{k}, k \in \mathbb{N}$.

P1. Стороны прямоугольников параллельны осям выбранной системы координат и отношение длины большей стороны $P_{k} \kappa$ длине меньшей стороны не превосходит трех.

P2. Каждая точка плоскости попадает во внутренность не более чем четырех прямоугольников $P_{k}$.

P3. Диаметры прямоугольников $P_{k}$ не превосходят 3 и $0 \notin \bigcup P_{k}$.

Предположим также, что существует целая функиия $F(\lambda), F(0)=1$, делящаяся на функиию $f(\lambda)$ и удовлетворяющая оценке

$$
\ln |F(\lambda)| \leqslant H(\lambda), \quad \lambda \in \mathbb{C},
$$

с некоторой липиичевой функцией $H$ :

$$
|H(\lambda)-H(z)| \leqslant \sigma|\lambda-z|, \quad \lambda, z \in \mathbb{C} .
$$

Тогда функция $f$ представляется в виде произведения цельх функиий $f_{1}, f_{2}$ так, что для любого $\lambda$ и $p, p>1$, выполняется соотношение

$$
\begin{aligned}
|\ln | f_{1}(\lambda)|-\ln | f_{2}(\lambda)|| \leqslant \frac{C_{0}}{p} & {[H(\lambda)-\ln |F(\lambda)|] } \\
& +C_{1} \ln ^{+} \frac{1}{\rho(\lambda)}+C_{2} \ln (|\lambda|+1)+C_{3} e^{p}, \quad \lambda \in \mathbb{C},
\end{aligned}
$$


где $\rho(\lambda)$ - расстояние от точки $\lambda$ до мнохсества нулей функции $f, C_{i}-$ постоянные, не зависящие от $р$.

Очевидно, целая функция $f$ с множеством нулей типа $\Lambda_{0}^{\prime \prime}$ удовлетворяет условиям теоремы 1 .

1.2. В этом пункте докажем вспомогательные теоремы для факторизации целых функций с множеством нулей типа $\Lambda_{0}^{\prime}$.

TEOPEMA 2. Пусть в квадрате

$$
P=\left\{1 \leqslant y \leqslant 1+2^{n} b, R \leqslant x<R+2^{n} b\right\},
$$

где $b \in[1 / 4,1 / 2]$, находится четное число точек $\lambda_{n}$, которые разбитын на пары $\lambda_{k}^{\prime}, \lambda_{k}^{\prime \prime}$ таким образом, что каждая пара $\lambda_{k}^{\prime}, \lambda_{k}^{\prime \prime}$ содержится в некотором прямоугольнике $P_{k} \subset P$, а система прямоугольников $\left\{P_{k}\right\}$ удовлетворяет условиям Р1, P2 в формулировке теоремы А и, дополнительно, следующим условиям.

P3'. Диаметры прямоугольников $P_{k}$ больие, чем 3 и $0 \notin \bigcup P_{k}$.

P4. Для любого индекса $k$ верна оценка

$$
\operatorname{diam} P_{k} \leqslant \frac{1}{2} \operatorname{dist}\left(P_{k}, 0\right)=\inf \left\{|z|, z \in P_{k}\right\}
$$

В верхней половине каждого прямоугольника $P_{k}$ въберем по одной произвольной точке $z_{k}$. Тогда существуют постоянные $C_{0}, B_{0}$, не зависящие от выбора точек $z_{k}$, такие, что для любого $x \in \mathbb{R}$ выполняется соотношение

$$
\begin{aligned}
& \sum_{k}|\ln | 1-\frac{x}{\lambda_{k}^{\prime}}|-\ln | 1-\frac{x}{\lambda_{k}^{\prime \prime}}\left|-\operatorname{Re} \frac{x\left(\lambda_{k}^{\prime}-\lambda_{k}^{\prime \prime}\right)}{\left(z_{k}-x\right) z_{k}}\right| \\
& \leqslant C_{0} \int_{P}\left|\frac{(2 w-x) x}{w^{2}(w-x)^{2}}\right| d v(w)+\delta(P, x) B_{0}\left[\ln \left(1+\frac{\operatorname{diam} P}{\operatorname{dist}(P, x)}\right)+\int_{P} \frac{1}{|w|^{2}} d v(w)\right],
\end{aligned}
$$

әде $\delta(P, x)$ обозначает число равное 1, если $\operatorname{diam} P>\operatorname{dist}(P, x) / 2$, и равное 0 в противном случае.

Доказательство этой теоремы будет следовать из следующих двух лемм. При фиксированном $x \in \mathbb{R}$ через $I_{1}(x)$ обозначим множество индексов $k$, для которых

$$
\operatorname{diam} P_{k} \leqslant \frac{1}{2} \operatorname{dist}\left(P_{k}, x\right)
$$

а через $I_{2}(x)$ все остальные индексы, т.е. такие, для которых

$$
\operatorname{diam} P_{k}>\frac{1}{2} \operatorname{dist}\left(P_{k}, x\right)
$$


Лемма 1. Для любого $x \in \mathbb{R}$ имеет место неравенство

$$
\sum_{k \in I_{1}(x)}|\ln | 1-\frac{x}{\lambda_{k}^{\prime}}|-\ln | 1-\frac{x}{\lambda_{k}^{\prime \prime}}\left|-\operatorname{Re} \frac{x\left(\lambda_{k}^{\prime}-\lambda_{k}^{\prime \prime}\right)}{\left(z_{k}-x\right) z_{k}}\right| \leqslant C_{0} \int_{P}\left|\frac{(2 w-x) x}{w^{2}(w-x)^{2}}\right| d v(w) .
$$

ДокАЗАТЕЛЬСТво. По теореме о среднем значении имеем

$$
\ln \left|1-\frac{x}{\lambda_{k}^{\prime}}\right|-\ln \left|1-\frac{x}{z_{k}}\right|-\operatorname{Re} \frac{x\left(\lambda_{k}^{\prime}-z_{k}\right)}{\left(z_{k}-x\right) z_{k}}=-\operatorname{Re} \frac{x\left(2 z_{k}^{\prime}-x\right)\left(\lambda_{k}^{\prime}-z_{k}\right)^{2}}{2\left(z_{k}^{\prime}\left(z_{k}^{\prime}-x\right)\right)^{2}},
$$

где $z_{k}^{\prime}$ - некоторая точка, лежащая на отрезке, соединяющем $\lambda_{k}^{\prime} \mathrm{c} z_{k}$. Аналогичная формула выполняется для точки $\lambda_{k}^{\prime \prime}$. Вычитая одно равенство из другого, получим оценку

$$
|\ln | 1-\frac{x}{\lambda_{k}^{\prime}}|-\ln | 1-\frac{x}{\lambda_{k}^{\prime \prime}}\left|-\operatorname{Re} \frac{x\left(\lambda_{k}^{\prime}-\lambda_{k}^{\prime \prime}\right)}{\left(z_{k}-x\right) z_{k}}\right| \leqslant \max _{w \in P_{k}}\left|\frac{(2 w-x) x}{w^{2}(w-x)^{2}}\right|\left(\operatorname{diam} P_{k}\right)^{2} .
$$

Для $k \in I_{1}$ с учетом условия $\mathrm{P} 4$ имеем оценку

$$
\max _{w \in P_{k}}\left|\frac{(2 w-x) x}{w^{2}(w-x)^{2}}\right| \leqslant C_{1}\left|\frac{\left(2 \zeta_{k}-x\right) x}{\zeta_{k}^{2}\left(\zeta_{k}-x\right)^{2}}\right|
$$

где $C_{1}$ - абсолютная постоянная, $\zeta_{k}$ - геометрический центр прямоугольника $P_{k}$. В правой части этого неравенства стоит функция, субгармоническая по переменной $\zeta_{k}$. По свойству $\mathrm{P} 4$ прямоугольник $P_{k}$ содержит круг с центром в точке $\zeta_{k}$ и радиуса $\operatorname{diam} P_{k} / 4:=d_{k} / 4$. Пользуясь свойством субгармонических функций получим оценку

$$
\max _{w \in P_{k}}\left|\frac{(2 w-x) x}{w^{2}(w-x)^{2}}\right| \leqslant \frac{16 C_{1}}{\pi d_{k}^{2}} \int_{|z-\zeta| \leqslant d_{k} / 4}\left|\frac{(2 w-x) x}{w^{2}(w-x)^{2}}\right| d v(w) .
$$

Подставим эту оценку в неравенство (4) и просуммируем по всем $k \in I_{1}(x)$. Учитывая свойство Р2 системы прямоугольников $\left\{P_{k}\right\}$, получим утверждение леммы 1.

Лемма 2. Имеет место соотношение

$$
\begin{aligned}
\sum_{k}|\ln | 1 & -\frac{x}{\lambda_{k}^{\prime}}|-\ln | 1-\frac{x}{\lambda_{k}^{\prime \prime}}\left|-\operatorname{Re} \frac{x\left(\lambda_{k}^{\prime}-\lambda_{k}^{\prime \prime}\right)}{\left(z_{k}-x\right) z_{k}}\right| \\
\leqslant & C_{0} \int_{P}\left|\frac{(2 w-x) x}{w^{2}(w-x)^{2}}\right| d v(w) \\
& +\delta(P, x) B_{0}\left[\ln \left(1+\frac{\operatorname{diam} P}{\operatorname{dist}(P, x)}\right)+\int_{P} \frac{1}{|w|^{2}} d v(w)\right], \quad x \in \mathbb{R}
\end{aligned}
$$

где $B_{0}-$ абсолютная постоянная и $\delta(P, x)=1$, если

$$
\operatorname{diam} P>\frac{1}{2} \operatorname{dist}(P, x),
$$

$u \delta(P, x)=0$ в остальных случаях. 
ДоказАТЕЛЬСТво. В силу условия $\mathrm{P} 4$ имеет место вложение $I_{1}(0) \subset I_{2}(x)$. Неравенство

$$
\sum_{k \in I_{2}(x)}|\ln | \lambda_{k}^{\prime}|-\ln | \lambda_{k}^{\prime \prime}\left|-\operatorname{Re} \frac{\left(\lambda_{k}^{\prime}-\lambda_{k}^{\prime \prime}\right)}{z_{k}}\right| \leqslant C_{1} \int_{P} \frac{1}{|w|^{2}} d v(w)
$$

доказывается так же, как лемма 1 . Через $n_{1}(x, t)$ и $n_{2}(x, t)$ обозначим число точек $\lambda_{k}^{\prime}$ и, соответственно, $\lambda_{k}^{\prime \prime}, k \in I_{2}(x)$, лежащих в круге радиуса $t$ с центром в точке $x$. По условиям теоремы

$$
n_{1}(x, \operatorname{dist}(P, x))=n_{2}(x, \operatorname{dist}(P, x))=0 .
$$

$\mathrm{C}$ другой стороны, для $t \geqslant T:=\operatorname{diam} P+\operatorname{dist}(P, x)$ имеем равенство $n_{1}(x, t)=$ $n_{2}(x, t)$. Интегрированием по частям получим

$$
\begin{aligned}
\sum_{k \in I_{2}(x)}\left(\ln \left|\lambda_{k}^{\prime}\right|-\ln \left|\lambda_{k}^{\prime \prime}\right|\right) & =\int_{d(x)}^{T} \ln t d\left(n_{1}(x, t)-n_{2}(x, t)\right) \\
& =\int_{d(x)}^{T} \frac{n_{1}(x, t)-n_{2}(x, t)}{t} d t,
\end{aligned}
$$

где $d(x)=\operatorname{dist}(P, x)$. Ясно, что $\left|n_{1}(x, t)-n_{2}(x, t)\right|$ не превосходит количества прямоугольников $P_{k}, k \in I_{2}$, пересекающихся с окружностью $\{z:|z-x|=t\}$. В работе [8] доказано следующее утверждение (см. утверждение 1 в [8])

УТВЕРЖДЕНИЕ 1. Пусть $\left(P_{n}\right)$ - совокупность прямоугольников со сторонами, параллельныции осям некоторой системь координат.

Предположим, что

1. в прямоугольнике $P_{n}$ содержится некоторый круг радиуса $\alpha d_{n}$, где $d_{n}$ - диаметр $P_{n}$

2. каждая точка плоскости попадает во внутренность не более, чем $p$ прямоугольников $P_{n}$.

Тогда число прямоугольников, пересекающихся с кругом $\{z:|z-x| \leqslant t\}$ и имеющих диаметр больше, чем $b t, b>0$, не превосходит числа $B(\alpha, p, b)=$ $p(\alpha+1)^{2} / \alpha^{4} b^{2}+4 p$.

Если прямоугольник $P_{k}, k \in I_{2}(x)$, пересекается с окружностью $\{z:|z-x|=t\}$ и $\operatorname{diam} P_{k}<t / 3$, то

$$
\operatorname{dist}\left(P_{k}, x\right) \leqslant 2 \operatorname{diam} P_{k}<\frac{2 t}{3} .
$$

Это значит, что $\operatorname{diam} P_{k}>t / 3$. Полученное противоречие показывает, что диаметры этих прямоугольников больше, чем $t / 3$. Поэтому к прямоугольникам $P_{k}$, $k \in I_{2}(x)$, можно применить утверждение, полагая в нем $\alpha=1 / 3, p=4, b=1 / 3$. Таким образом, их количество не превосходит $145 \cdot 16$. Этой же константой оценивается и $\left|n_{1}(x, t)-n_{2}(x, t)\right|$. Из неравенства (6) получим

$$
\sum_{k \in I_{2}(x)}\left(\ln \left|\lambda_{k}^{\prime}-x\right|-\ln \left|\lambda_{k}^{\prime \prime}-x\right|\right) \leqslant B^{\prime} \ln \frac{T}{d(x)} .
$$


Так как в приведенных рассуждениях точки $\lambda_{k}^{\prime}, \lambda_{k}^{\prime \prime}$ выступают равноправно, то имеет место оценка

$$
\sum_{k \in I_{2}(x)}|\ln | \lambda_{k}^{\prime}-x|-\ln | \lambda_{k}^{\prime \prime}-x|| \leqslant B^{\prime} \ln \left(1+\frac{\operatorname{diam} P}{\operatorname{dist}(P, x)}\right) .
$$

Для $k \in I_{2}(x)$, учитывая выбор расположения точек $z_{k}$ (в верхней половине прямоугольника $\left.P_{k}\right)$, имеем $\left|\lambda_{k}^{\prime}-\lambda_{k}^{\prime \prime}\right| \leqslant \operatorname{diam} P_{k} \leqslant 8\left|z_{k}-x\right|$. Поэтому

$$
\operatorname{Re} \frac{\lambda_{k}^{\prime}-\lambda_{k}^{\prime \prime}}{z_{k}-x} \leqslant 8
$$

Прямоугольники $P_{k}$, как и весь квадрат $P$, лежат в кольце

$$
\{z: \operatorname{dist}(P, x) \leqslant|z-x| \leqslant \operatorname{dist}(P, x)+\operatorname{diam} P\} .
$$

Возьмем произвольное $t>0$ и рассмотрим прямоугольники $P_{k}, k \in I_{2}(x)$, пересекаюшиеся с кольцом $\{z: t \leqslant|z-x| \leqslant 2 t\}$. Количество тех из этих прямоугольников, которые пересекаются с окружностью $\{z:|z-x|=t\}$, не превосходит $B^{\prime}$. Если же прямоугольник не пересекается с этой окружностью, то $\operatorname{diam} P_{k}>\operatorname{dist}(P, x) / 2 \geqslant t / 2$, и этот прямоугольник пересекается с окружностью $\{z:|z-x|=3 t / 2\}$. Снова по утверждению 1 можем оценить количество таких прямоугольников. В результате мы получим, что число прямоугольников $P_{k}, k \in I_{2}(x)$, пересекающихся с кольцом $\{z: t \leqslant|x-z| \leqslant 2 t\}$, не превосходит некоторой абсолютной постоянной $B_{1}$. Тогда обшее число прямоугольников $P_{k}$, $k \in I_{2}(x)$, не превосходит величины

$$
\frac{B_{1}}{\ln 2} \ln \left(1+\frac{\operatorname{diam} P}{\operatorname{dist}(P, x)}\right)
$$

Поэтому, учитывая оценку (8), имеем

$$
\sum_{k \in I_{2}(x)}\left|\operatorname{Re} \frac{\lambda_{k}^{\prime}-\lambda_{k}^{\prime \prime}}{z_{k}-x}\right| \leqslant C \ln \left(1+\frac{\operatorname{diam} P}{\operatorname{dist}(P, x)}\right) .
$$

Отсюда и из соотношений (5), (7) вытекает утверждение леммы 2. Остается заметить, что если $\operatorname{diam} P \leqslant \operatorname{dist}(P, x) / 2$, то $I_{2}(x)=\varnothing$, вследствие чего и появляется множитель $\delta(P, x)$. Лемма 2 доказана.

Из утверждений лемм 1 и 2 следует утверждение теоремы 2.

ТЕорема 3. В условиях теоремъ 2 существует набор чисел $\varepsilon_{k}= \pm 1 u$ число $h \in \mathbb{C},|h| \leqslant 200 \operatorname{diam} P$, такие, что выполняется соотношение

$$
\begin{aligned}
& \left|\sum_{k} \varepsilon_{k}\left(\ln \left|1-\frac{x}{\lambda_{k}^{\prime}}\right|-\ln \left|1-\frac{x}{\lambda_{k}^{\prime \prime}}\right|\right)-\operatorname{Re} \frac{x h}{\left(z_{0}-x\right) z_{0}}\right| \\
& \quad \leqslant n C \int_{P}\left|\frac{(2 w-x) x}{w^{2}(w-x)^{2}}\right| d v(w)+\delta(P, x) B_{0}\left[\ln \left(1+\frac{\operatorname{diam} P}{\operatorname{dist}(P, x)}\right)+\int_{P} \frac{1}{|w|^{2}} d v(w)\right],
\end{aligned}
$$

где $z_{0}$ - геометрический чентр квадрата $P$, постоянные $C$ и $B$ не зависят om $R, b, n$. 
ДоКАЗАТЕЛЬСтво. Для $m=0,1, \ldots, n$ рассмотрим квадраты

$$
\begin{gathered}
Q_{s, l}^{(m)}=\left\{1+s b 2^{m} \leqslant y<(s+1) b 2^{m}, R+l b 2^{m} \leqslant x<R+(l+1) b 2^{m}\right\} \\
s=0,1, \ldots, 2^{n-m}, \quad l=0,1, \ldots, 2^{n-m} .
\end{gathered}
$$

В силу свойства Р 1 стороны каждого прямоугольника $P_{k}$ неменьше, чем $\operatorname{diam} P_{k} / 4$. Поэтому в верхней половине каждого прямоугольника $P_{k}$ содержится по меньшей мере один квадрат $Q_{s, l}^{(m)}$ с длиной стороны не меньше, чем $\operatorname{diam} P_{k} / 16$. Для каждого прямоугольника $P_{k}$ выберем по одному такому квадрату. Каждый из квадратов $Q_{s, l}^{(m)}$ в силу условия P2 может быть выбран не более чем четыре раза. Если квадрат $Q_{s, l}^{(m)}$ выбран для прямоугольников $P_{k_{i}}, 0<i \leqslant 4$, то через $h_{s, l}^{(m)}$ обозначим сумму разностей $\left(\lambda_{k}^{\prime}-\lambda_{k}^{\prime \prime}\right)$. Если же этот квадрат не выбран ни для одного прямоугольника, то $h_{s, l}^{(m)}$ положим равньм нулю. Заметим, что всегда вьполняется оценка

$$
\left|h_{s, l}^{(m)}\right| \leqslant \frac{1}{4} \operatorname{diam} Q_{s, l}^{(m)} .
$$

В наших обозначениях имеет место равенство

$$
Q_{s, l}^{(m)}=Q_{2 s, 2 l}^{(m-1)} \cup Q_{2 s, 2 l+1}^{(m-1)} \cup Q_{2 s+1,2 l}^{(m-1)} \cup Q_{2 s, 2+1}^{(m-1)} .
$$

Лемма 3. Для любого набора чисел $z_{1}, \ldots, z_{n}$ существуют числа $\varepsilon_{k}= \pm 1$ такие, что

$$
\left|\varepsilon_{1} z_{1}+\cdots+\varepsilon_{n} z_{n}\right| \leqslant \sqrt{2} \max _{i=1, \ldots, n}\left|z_{i}\right| .
$$

ДоКАЗАТЕЛЬСТво проведем методом математической индукции. Пусть $n=2$. Тогда если $\left|z_{1} \pm z_{2}\right|>\max \left(\left|z_{1}\right|,\left|z_{2}\right|\right)$, то

$$
4\left(\max \left(\left|z_{1}\right|,\left|z_{2}\right|\right)\right)^{2}<\left|z_{1}+z_{2}\right|^{2}+\left|z_{1}-z_{2}\right|^{2}=2\left(\left|z_{1}\right|^{2}+\left|z_{2}\right|^{2}\right) \leqslant\left(\max \left(\left|z_{1}\right|,\left|z_{2}\right|\right)\right)^{2} .
$$

Следовательно, одно из чисел $\left|z_{1}+z_{2}\right|$ и $\left|z_{1}-z_{2}\right|$ не превосходит $\sqrt{2} \max \left(\left|z_{1}\right|,\left|z_{2}\right|\right)$. Векторы $\pm z_{i}, i=1, \ldots, n$, разбивают плоскость на $2 n$ углов (считаем, что $z_{i} \neq 0$ ). Если $n>2$, то один из этих углов меньше $\pi / 3$. Пусть, например, это угол между $z_{1}$ и $z_{2}$. Тогда $\left|z_{1}-z_{2}\right| \leqslant \max \left(\left|z_{1}\right|,\left|z_{2}\right|\right)$. Для чисел $z_{1}-z_{2}, z_{3}, \ldots, z_{n}$ по допушению индукции сушествуют числа $\varepsilon_{k}^{\prime}, k=1, \ldots, n-1$, такие, что

$$
\left|\varepsilon_{1}^{\prime}\left(z_{1}-z_{2}\right)+\varepsilon_{2}^{\prime} z_{3}+\cdots+\varepsilon_{n-1}^{\prime} z_{n}\right| \leqslant \sqrt{2} \max _{i}\left(\left|z_{1}-z_{2}\right|,\left|z_{i}\right|\right) .
$$

Тогда система $\varepsilon_{1}=\varepsilon_{1}^{\prime}, \varepsilon_{2}=-\varepsilon_{1}^{\prime}, \varepsilon_{i}=\varepsilon_{i-1}, i=3, \ldots, n$, удовлетворяет условиям леммы 3.

Теперь мы можем описать выбор чисел $\varepsilon_{k}$ из утверждения теоремы 3. Сначала рассуждения проведем при одном дополнительном условии:

$(*)$ если $h_{s, l}^{(m)} \neq 0$, то для всех $Q_{i, j}^{(k)}$, лежащих в квадрате $Q_{s, l}^{(m)}$, соответствующие числа $h_{i, j}^{(k)}$ равны нулю. 
Множество всех $h_{s, l}^{(m)}$ обозначим через $H$. Пусть $h_{s, l}^{(1)}=0$. Для четырех комплексных чисел $h_{i, j}^{(0)}, i=2 s, 2 s+1, j=2 l, 2 l+1$, по лемме 3 выберем числа $\sigma_{i, j}^{(0)}= \pm 1$ так, чтобы выполнялась оценка

$$
\left|\sum \sigma_{i, j}^{(0)} h_{i, j}^{(0)}\right| \leqslant \sqrt{2} \max \left|h_{i, j}^{(0)}\right|
$$

и переопределим $h_{s, l}^{(1)}$, положив его равньм

$$
\sum \sigma_{i, j}^{(0)} h_{i, j}^{(0)}
$$

Произведя эту процедуру со всеми ненулевьми $h_{s, l} h^{(1)}$, мы получим новое множество чисел $h_{s, l}^{(m)}, m \geqslant 1$, удовлетворяюших оценке (9). Обозначим это множество через $H^{(1)}$. Очевидно, $H^{(1)}$ удовлетворяет условию $(*)$. Далее проделаем эту процедуру с нулевьми векторами $h_{s, l}^{(2)} \in H^{(1)}$ и получим новое множество $H^{(2)}$, состоящее из чисел $h_{s, l}^{(m)}, m \geqslant 2$, удовлетворяющих оценке (9) и условию $(*)$. Продолжая этот процесс, через $n$ шагов мы получим множество $H^{(n)}$, состояшее из одного числа $h_{0,0}^{(n)}$. По построению

$$
h_{0,0}^{(n)}=\sum_{H} \varepsilon_{s, l}^{(m)} h_{s, l}^{(m)},
$$

где $\sum_{H}$ означает суммирование по всем $h_{s, l}^{(m)} \in H$, а числа $\varepsilon_{s, l}^{(m)}$ получаются как произведение некоторых $\sigma_{i, j}^{(k)}$. Например, если $h_{0,0}^{(0)} \neq 0$, то по условию все числа $h_{0,0}^{(m)} \in H, m=1, \ldots, n$, равны нулю и $\varepsilon_{0,0}^{(0)}=\sigma_{0,0}^{(0)} \sigma_{0,0}^{(1)} \cdots \sigma_{0,0}^{(n)}$. С другой стороны, каждое ненулевое число $h_{s, l}^{(m)} \in H$, является суммой некоторых (не более четырех) разностей $\left(\lambda_{k}^{\prime}-\lambda_{k}^{\prime \prime}\right)$, и каждая разность $\left(\lambda_{k}^{\prime}-\lambda_{k}^{\prime \prime}\right)$ входит как слагаемое в одно и только в одно число $h_{s, l}^{(m)}$. Следовательно, для некоторых $\varepsilon_{k}= \pm 1$ имеет место равенство

$$
h_{0,0}^{(m)}=\sum \varepsilon_{k}\left(\lambda_{k}^{\prime}-\lambda_{k}^{\prime \prime}\right)
$$

Докажем, что числа $\varepsilon_{\boldsymbol{k}}$ в соотношении $(10)$ и $h=h_{0,0}^{(n)}$ удовлетворяют условиям теоремы 3. Для этого в силу утверждения теоремы 2 достаточно доказать, что вьполняется соотношение

$$
\left|\sum_{k} \varepsilon_{k} \operatorname{Re} \frac{x\left(\lambda_{k}^{\prime}-\lambda_{k}^{\prime \prime}\right)}{\left(z_{k}-x\right) z_{k}}-\operatorname{Re} \frac{x h}{\left(z_{0}-x\right) z_{0}}\right| \leqslant n C \int_{P}\left|\frac{(2 w-x) x}{w^{2}(w-x)^{2}}\right| d v(w) .
$$

В выборе точек $z_{k}$ у нас есть определенньй произвол. Эти точки должны лежать в верхних половинках прямоугольников $P_{k}$. Возьмем в качестве $z_{k}$ геометрический центр $z_{s, l}^{(m)}$ квадрата $Q_{s, l}^{(m)}$, выбранного для прямоугольника $P_{k}$. Тогда, очевидно,

$$
\sum_{k} \varepsilon_{k} \operatorname{Re} \frac{x\left(\lambda_{k}^{\prime}-\lambda_{k}^{\prime \prime}\right)}{\left(z_{k}-x\right) z_{k}}=\sum_{H} \varepsilon_{s, l}^{(m)} \operatorname{Re} \frac{x h_{s, l}^{(m)}}{\left(z_{s, l}^{(m)}-x\right) z_{s, l}^{(m)}},
$$


где $\sum$ означает суммирование по всем $h_{s, l}^{(m)}$ из $H$. Оценка (11) будет следовать из соотношения

$$
\begin{array}{r}
\left|\sum_{H} \varepsilon_{s, l}^{(m)} \operatorname{Re} \frac{x h_{s, l}^{(m)}}{\left(z_{s, l}^{(m)}-x\right) z_{s, l}^{(m)}}-\operatorname{Re} \frac{x h_{0,0}^{(n)}}{\left(z_{0,0}^{(n)}-x\right) z_{0,0}^{(n)}}\right| \\
\leqslant n C \int_{P}\left|\frac{(2 w-x) x}{w^{2}(w-x)^{2}}\right| d v(w) .
\end{array}
$$

Вернемся к конструкции множеств $H^{(k)}, k=1, \ldots, n$. Пусть $h_{s, l}^{(1)} \in H^{(1)} \backslash H$. По построению

$$
h_{s, l}^{(1)}=\sum \sigma_{i, j}^{(0)} h_{i, j}^{(0)}
$$

где суммирование ведется по $i=2 s, 2 s+1, j=2 l, 2 l+1$. По теореме о среднем имеем

$$
\sigma_{i, j}^{(0)} \operatorname{Re} \frac{x h_{i, j}^{(0)}}{\left(z_{i, j}^{(0)}-x\right) z_{i, j}^{(0)}}=\operatorname{Re} \frac{x h_{i, j}^{(0)} \sigma_{i, j}^{(0)}}{\left(z_{i, j}^{(0)}-x\right) z_{i, j}^{(0)}}+\operatorname{Re} \frac{\sigma_{i, j}^{(0)} x h_{i, j}^{(0)}\left(z_{i, j}^{(0)}-z_{s, l}^{(1)}\right)}{(w-x)^{2} w^{2}}
$$

где $w$ - некоторая точка на отрезке, соединяющем точки $z_{i, j}^{(0)}$ и $z_{s, l}^{(1)}$. Суммируя эти равенства и учитывая, что $\left|z_{i, j}^{(0)}-z_{s, l}^{(1)}\right| \leqslant \operatorname{diam} Q_{s, l}^{(1)} / 4$, получим оценку (см. (9))

$$
\begin{aligned}
& \left|\sum_{i, j} \sigma_{i, j}^{(0)} \operatorname{Re} \frac{x h_{i, j}^{(0)}}{\left(z_{i, j}^{(0)}-x\right) z_{i, j}^{(0)}}-\operatorname{Re} \frac{x h_{s, l}^{(1)}}{\left(z_{s, l}^{(1)}-x\right) z_{s, l}^{(1)}}\right| \\
& \quad \leqslant \max _{w}\left|\frac{(2 w-x) x}{w^{2}(w-x)^{2}}\right| \operatorname{diam} Q_{s, l}^{(1)} \operatorname{diam} Q_{i, j}^{(0)} \cdot 48 \\
& \quad=\max _{w}\left|\frac{(2 w-x) x}{w^{2}(w-x)^{2}}\right| \operatorname{diam} Q_{s, l}^{(1)} \cdot 24 .
\end{aligned}
$$

При этом максимум можно брать по квадрату с вершинами в точках $z_{i, j}^{(0)}$. Учитывая условие Р 4 , получим, что сушествует постоянная $B_{0}$ такая, что

$$
\max _{w}\left|\frac{(2 w-x)}{w^{2}(w-x)^{2}}\right| \leqslant B_{0}\left|\frac{\left(2 z_{s, l}^{(1)}-x\right)}{\left(z_{s, l}^{(1)}\left(z_{s, l}^{(1)}-x\right)\right)^{2}}\right|
$$

В правой части этого неравенства стоит функция, субгармоническая по переменной $z_{s, l}^{(1)}$ в $P$, и круг с центром в точке $z_{s, l}^{(1)}$ и радиуса $\operatorname{diam} Q_{s, l}^{(1)} / 2$ лежит в квадрате $Q_{s, l}^{(1)}$. Поэтому

$$
\max _{w}\left|\frac{(2 e-x)}{w^{2}(w-x)^{2}}\right| \leqslant \frac{2 B_{0}}{\pi}\left(\operatorname{diam} Q_{s, l}^{(1)}\right)^{2} \int_{Q_{s, l}^{(1)}}\left|\frac{(2 w-x) x}{w^{2}(w-x)^{2}}\right| d v(w)
$$


Подставим эту оценку в неравенство (12) и просуммируем по всем $h_{s, l}^{(1)} \in H^{(1)} \backslash H$. В результате получим соотношение

$$
\begin{array}{r}
\left|\sum_{H} \varepsilon_{s, l}^{(m)} \operatorname{Re} \frac{x h_{s, l}^{(m)}}{\left(z_{s, l}^{(m)}-x\right) z_{s, l}^{(m)}}-\sum_{H^{(1)}} \varepsilon_{s, l}^{(m)} \operatorname{Re} \frac{x h_{s, l}^{(m)}}{\left(z_{s, l}^{(m)}-x\right) z_{s, l}^{(m)}}\right| \\
\leqslant \frac{2 B_{0}}{\pi} \int_{P}\left|\frac{(2 w-x) x}{w^{2}(w-x)^{2}}\right| d v(w) .
\end{array}
$$

Совершенно также оценивается разность между $\sum_{H^{(k)}}$ и $\sum_{H^{(k+1)}}$. Поскольку $H^{(n)}=\left\{h_{0,0}^{(n)}\right\}$, то в итоге мы получим требуемую оценку (12), которая доказывает теорему 3 при дополнительном условии (*). Для того чтобы избавиться от этого условия, разобьем множество $H$ на четыре непересекающиеся части $H_{i}$, каждая из которых удовлетворяет условию $(*)$. Если это будет сделано, то доказав утверждение теоремы 3 для каждого из множеств $H_{i}$ по отдельности, мы получим доказательство в общем случае. Через $H_{1}$ обозначим множество чисел $h_{s, l}^{(m)} \in H$, для которых $h_{i, j}^{(k)}=0$ всякий раз, когда $Q_{i, j}^{(k)} \subset Q_{s, l}^{(m)}$. Через $H_{2}$ обозначим отличные от нуля числа $h_{s, l}^{(m)} \in H \backslash H_{1}$, для которых все $h_{i, j}^{(k)} \in H \backslash H_{1}$ равны нулю, если $Q_{i, j}^{(k)} \subset Q_{s, l}^{(m)}$. Аналогично определим $H_{3} \subset H_{1} \backslash H_{2}$. Очевидно, $H_{3}, H_{1}, H_{2}$, дополненные нулями до полного набора, удовлетворяют условию $(*)$. Докажем, что $H_{4}=H \backslash\left(H_{1} \cup H_{2} \cup H_{3}\right)$ также удовлетворяет условию $(*)$. Действительно, если $h_{s, l}^{(m)} \neq 0, h_{s, l}^{(m)} \in H_{4}$ и найдется $h_{i, j}^{(k)} \in H_{4}, h_{i, j}^{(k)} \neq 0$, причем $Q_{i, j}^{(k)} \subset Q_{s, l}^{(m)}$, то $h_{i, j}^{(k)} \notin H_{3}$. По построению это значит, что найдется $h_{m, r}^{(t)} \neq 0, h_{m, r}^{(t)} \in H_{3}$ и $Q_{m, r}^{(t)} \subset Q_{i, j}^{(k)}$. Продолжая эти рассуждения, мы придем к существованию цепочки

$$
Q_{x, y}^{(z)} \subset Q_{u, v}^{(p)} \subset Q_{m, r}^{(t)} \subset Q_{i, j}^{(k)} \subset Q_{s, l}^{(m)}
$$

причем

$$
h_{x, y}^{(z)} \neq 0, \quad h_{m, r}^{(t)} \neq 0, \quad h_{i, j}^{(k)} \neq 0, \quad h_{s, l}^{(m)} \neq 0
$$

т.е. все эти квадраты были выбраны для некоторых $P_{k}$. Отсюда следует, что квадрат $Q_{x, y}^{(z)}$ лежит не менее чем в пяти прямоугольниках, что противоречит свойстBy $\mathrm{P} 2$.

Теорема 3 доказана.

1.3. Перейдем непосредственно к вопросу о факторизации целых функций с множеством нулей типа $\Lambda_{0}^{\prime}$.

Напомним, что для функции

$$
a(x)=\exp (a \sqrt{\ln (x+1)}), \quad x \geqslant 0
$$

определили последовательность $x_{n}$ по формуле $x_{0}=0$ и

$$
a\left(x_{n}\right)=x_{n}-x_{n-1}+1, \quad n=1,2, \ldots
$$


Целая функция $f$ в каждом из квадратов

$$
Q_{n}=\left\{z=x+i y, x_{n-1} \leqslant x<x_{n}, 1<y \leqslant x_{n}-x_{n-1}+1\right\}
$$

и $-Q_{n}, \pm Q_{n}^{\prime}$ имеет четное число нулей, разбитых на пары $\lambda_{k}^{\prime}, \lambda_{k}^{\prime \prime}$. Каждая пара покрыта прямоугольником $P_{k}$ и система прямоугольников удовлетворяет условиям P1, P2 теоремы А и Р3' в условии теоремы 2. Не уменьшая обшности можно считать выполненным также условие Р 4 . Возьмем один из квадратов $Q_{n}$. Этот квадрат представим в виде, описанном в теореме 2. Достаточно положить

$$
n=n_{m}=\frac{\ln \left(x_{m}-x_{m-1}\right)}{\ln 2}+2, \quad b=2^{\alpha-2}, \quad R=x_{m-1},
$$

где $[x]$ обозначает целую часть $x$ и $\alpha<1$. К нулям функции $f$, лежашим в квадрате $Q_{m}$, применим теоремы 2 и 3 . Из них следует, что существуют числа $\varepsilon_{k}= \pm 1$ такие, что для любого $x \in \mathbb{R}$ выполняется соотношение

$$
\begin{aligned}
& \left|\sum_{k} \varepsilon_{k}\left(\ln \left|1-\frac{x}{\lambda_{k}^{\prime}}\right|-\ln \left|1-\frac{x}{\lambda_{k}^{\prime \prime}}\right|\right)-\operatorname{Re} \frac{x h_{m}}{\left(z_{m}-x\right) z_{m}}\right| \\
& \leqslant C n_{m} \int_{Q_{m}}\left|\frac{(2 w-x) x}{w^{2}(w-x)^{2}}\right| d v(w) \\
& \quad+\delta\left(Q_{m}, x\right) B\left[\ln \left(1+\frac{\operatorname{diam} Q_{m}}{\operatorname{dist}\left(Q_{m}, x\right)}\right)+\int_{Q_{m}} \frac{1}{|w|^{2}} d v(w)\right],
\end{aligned}
$$

где $z_{m}$ - геометрический центр $Q_{m}$ и

$$
\left|h_{m}\right| \leqslant 200 \operatorname{diam} Q_{m}
$$

Если $\varepsilon_{k}=-1$, то поменяем местами точки $\lambda_{k}^{\prime}$ и $\lambda_{k}^{\prime \prime}$ и будем считать выполненньм соотношение

$$
\begin{aligned}
& \left|\sum_{k}\left(\ln \left|1-\frac{x}{\lambda_{k}^{\prime}}\right|-\ln \left|1-\frac{x}{\lambda_{k}^{\prime \prime}}\right|\right)-\operatorname{Re} \frac{x h_{m}}{\left(z_{m}-x\right) z_{m}}\right| \\
& \leqslant C n_{m} \int_{Q_{m}}\left|\frac{(2 w-x) x}{w^{2}(w-x)^{2}}\right| d v(w) \\
& \quad+\delta\left(Q_{m}, x\right) B\left[\ln \left(1+\frac{\operatorname{diam} Q_{m}}{\operatorname{dist}\left(Q_{m}, x\right)}\right)+\int_{Q_{m}} \frac{1}{|w|^{2}} d v(w)\right],
\end{aligned}
$$

где $C, B$ - постоянные, не зависящие от $m$. Такие же оценки выполняются для квадратов $-Q_{m}, \pm Q_{m}^{\prime}$.

ТЕОРемА 4. Пусть целая функция $f$ удовлетворяет условиям основной теоремы. Нули функиии $f$ находятся в квадратах $\pm Q_{m}, \pm Q_{m}^{\prime}$, причем в каждом квадрате находится четное число нулей и они разбиты на пары $\lambda_{k}^{\prime}, \lambda_{k}^{\prime \prime}$. Каждая пара покрыта прямоугольником $P_{k}$ и система прямоугольников $P_{k}$ удовлетворяет условиям $\mathrm{P} 1, \mathrm{P} 2, \mathrm{P} 3^{\prime}, \mathrm{P} 4$ в формулировке теоремы 2. Тогда для всех $x \in \mathbb{R}$ выполняется соотночение

$$
\left|\sum_{k}\left(\ln \left|1-\frac{x}{\lambda_{k}^{\prime}}\right|-\ln \left|1-\frac{x}{\lambda_{k}^{\prime \prime}}\right|\right)\right| \leqslant C \ln (|x|+1) .
$$


ДОКАЗАТЕЛЬСТВО проведем на основе соотношения (13).

Лемма 4. Имеет место оценка

$$
\sum_{m=1}^{\infty}\left|\frac{x h_{m}}{\left(z_{m}-x\right) z_{m}}\right| \leqslant C \ln (|x|+1) .
$$

ДОКАЗАТЕЛЬСТво ЛЕММЫ 4. По ПостроенИю

$$
\left|h_{m}\right| \leqslant 200 \operatorname{diam} Q_{m} \leqslant 300\left(x_{m}-x_{m-1}\right)
$$

(см. теорему 3$)$. Через $x_{m}^{\prime}$ обозначим $\left(x_{m}+x_{m-1}\right) / 2$. Тогда

$$
\begin{aligned}
\sum_{m=1}^{\infty}\left|\frac{x h_{m}}{\left(z_{m}-x\right) z_{m}}\right| & \leqslant x C_{1} \sum_{m=1}^{\infty} \frac{x_{m}-x_{m-1}}{\left(\left|x_{m}^{\prime}-x\right|+a\left(x_{m}\right)\right)\left(x_{m}^{\prime}+a\left(x_{m}\right)\right)} \\
& \leqslant C_{2} x \sum_{m=1}^{\infty} \int_{x_{m-1}}^{x_{m}} \frac{d t}{(|t-x|+a(t))(t+a(t))}
\end{aligned}
$$

Учитывая, что $a(t) \geqslant 1$, отсюда получим оценку

$$
\begin{aligned}
\sum_{m=1}^{\infty}\left|\frac{x h_{m}}{\left(z_{m}-x\right) z_{m}}\right| & \leqslant C_{2} x \int_{0}^{\infty} \frac{d t}{(|t-x|+1)(t+1)} \\
& \leqslant C_{2}\left(1+\frac{|x|}{|x|+2}\right) \ln (|x|+1)
\end{aligned}
$$

Лемма 4 доказана.

ЛЕмма 5. Для любого $x \in \mathbb{R}$ выполняется неравенство

$$
\sum_{m=1}^{\infty} n_{m} \int_{Q_{m}}\left|\frac{(2 w-x) x}{w^{2}(w-x)^{2}}\right| d v(w) \leqslant \mathrm{const} \cdot \ln (|x|+1) .
$$

ДоКАЗАТЕЛЬСТво. По определению чисел $n_{m}$ имеем оценку

$$
n_{m} \leqslant \text { const } \cdot \ln a\left(x_{m}\right) \leqslant \mathrm{const} \cdot \ln a(t), \quad x_{m-1} \leqslant x \leqslant x_{m},
$$

поэтому

$$
\sum_{m=1}^{\infty} n_{m} \int_{Q_{m}}\left|\frac{(2 w-x) x}{w^{2}(w-x)^{2}}\right| d v(w) \leqslant \int_{G} \frac{|2 w-x||x| \ln a(\operatorname{Re} w)}{|w-x|^{2}|w|^{2}} d v(w)
$$

где

$$
G=\{1 \leqslant \operatorname{Re} w \leqslant \infty, 1 \leqslant \operatorname{Im} w \leqslant A a(\operatorname{Re} w)\}
$$


и число $A$ определено во введении. Воспользуемся следующими элементарными оценками

$$
\begin{array}{ll}
\frac{|2 w-x||x| \ln a(\operatorname{Re} w)}{|w-x|^{2}|w|^{2}} \leqslant \frac{C \ln a(|x|)}{|w|^{2}}, & w \in G, \quad 1 \leqslant \operatorname{Re} w \leqslant x / 2, \\
\frac{|2 w-x||x| \ln a(\operatorname{Re} w)}{|w-x|^{2}|w|^{2}} \leqslant \frac{C \ln a(|x|)}{|w-x|^{2}}, & w \in G, \quad x / 2 \leqslant \operatorname{Re} w \leqslant 2 x, \\
\frac{|2 w-x||x| \ln a(\operatorname{Re} w)}{|w-x|^{2}|w|^{2}} \leqslant \frac{C \ln a(\operatorname{Re} w)}{|w|^{2}}, & w \in G, \quad 2 x \leqslant \operatorname{Re} w,
\end{array}
$$

где $C>0$ - некоторая постоянная. По первой из этих оценок, переходя к полярным координатам, получим

$$
\begin{aligned}
& \int_{G \cap\{1 \leqslant \operatorname{Re} w \leqslant x / 2\}} \frac{|2 w-x||x| \ln a(\operatorname{Re} w)}{|w-x|^{2}|w|^{2}} d v(w) \\
& \leqslant C_{1} \ln a(x) \int_{1}^{x / 2} \frac{a(r)}{r^{2}} d r \leqslant \operatorname{const} \cdot \ln a(x), \quad w \in G, \quad 1 \leqslant \operatorname{Re} w \leqslant x / 2,
\end{aligned}
$$

так как $a(r)=O\left(r^{t}\right)$ для любого $t>0$. Аналогично оценивается интеграл по множеству $G \cap\{2 x \leqslant \operatorname{Re} w\}$. По второму из вышеприведенных неравенств получим

$$
\begin{aligned}
\int_{G \cap\{x / 2 \leqslant \operatorname{Re} w \leqslant 2 x\}} \frac{|2 w-x||x| \ln a(\operatorname{Re} w)}{|w-x|^{2}|w|^{2}} d v(w) \\
\leqslant C_{1} \ln a(x) \int_{1}^{A a(2 x)} \int_{x / 2}^{2 x} \frac{d t d s}{(t-x)^{2}+s^{2}} \\
=C_{1} \ln a(x) \int_{1}^{A a(2 x)} \frac{1}{s} \int_{x / 2 s}^{x / s} \frac{d \tau}{\tau^{2}+1} d s \\
\leqslant C_{1} \pi \ln a(x) \ln a(2 x) \leqslant C_{2}(\ln a(x))^{2} \leqslant C_{3} \ln (|x|+1) .
\end{aligned}
$$

Лемма 5 доказана.

Совершенно так же доказываются утверждения лемм 4 и 5 для квадратов $-Q_{m}$ и $\pm Q_{m}^{\prime}$. Из этих оценок и из неравенства (13) получим соотношение

$$
\begin{aligned}
& \left|\sum_{k}\left(\ln \left|1-\frac{x}{\lambda_{k}^{\prime}}\right|-\ln \left|1-\frac{x}{\lambda_{k}^{\prime \prime}}\right|\right)\right| \\
& \leqslant C \ln (|x|+1)+\sum_{m} \delta\left(Q_{m}, x\right) B\left[\ln \left(1+\frac{\operatorname{diam} Q_{m}}{\operatorname{dist}\left(Q_{m}, x\right)}\right)+\int_{Q_{m}} \frac{1}{|w|^{2}} d v(w)\right],
\end{aligned}
$$

где слева суммирование ведется по всем индексам $m$. Напомним, что величина $\delta\left(Q_{m}, x\right)$ отлична от нуля лишь в том случае, когда $\operatorname{diam} Q_{m}$ больше, чем $\operatorname{dist}\left(Q_{m}, x\right) / 2$. Этому условию могут удовлетворить не более 16 квадратов $Q_{m}$, ближайших к точке $x$. При этом для этих квадратов выполняется оценка

$$
\ln \left(1+\frac{\operatorname{diam} Q_{m}}{\operatorname{dist}\left(Q_{m}, x\right)}\right)+\int_{Q_{m}} \frac{1}{|w|^{2}} d v(w) \leqslant \text { const } \cdot \ln a(|x|) \leqslant \text { const } \cdot \ln (|x|+1) .
$$

Отсюда и из оценки (14) следует утверждение теоремы 4. 
Теорема 5. В условиях теоремь 4 челая функиия $f$ может быть представлена как произведение целых функиий $f_{1}, f_{2}$ так, что для любого $\varepsilon>0$ выполняются соотношения

$$
\begin{gathered}
|\ln | f_{1}(x)|-\ln | f_{2}(x)|| \leqslant \mathrm{const} \cdot \ln (|x|+1), \quad x \in \mathbb{R}, \\
|\ln | f_{1}\left(r e^{i \varphi}\right)|-\ln | f_{2}\left(r e^{i \varphi}\right)|| \leqslant C(\varphi, \varepsilon) r^{\varepsilon}, \quad r>r(\varphi, \varepsilon) .
\end{gathered}
$$

ДокАЗАТЕльСтво. Целая функция $f$ удовлетворяет условиям основной теоремы. Если через $n_{f}(t)$ обозначим число нулей функции $f$ в круге радиуса $t$ с центром в начале координат, а через $n_{F}(t)$ - число нулей функции $F$ в том же круге, то $n_{f}(t) \leqslant n_{F}(t)$ и по формуле Иенсена получим оценку

$$
\begin{aligned}
\int_{0}^{r} \frac{n_{f}(t)}{t} d t \leqslant \int_{0}^{r} \frac{n_{F}(t)}{t} d t & \leqslant \frac{1}{\pi} \int_{0}^{2 \pi} \ln \left|F\left(r e^{i \varphi}\right)\right| d \varphi \\
& \leqslant \frac{1}{\pi} \int_{0}^{2 \pi} H\left(r e^{i \varphi}\right) d \varphi \leqslant \sigma r+H(0) .
\end{aligned}
$$

Отсюда вытекает оценка

$$
n_{f}(t) \leqslant \int_{t}^{e t} \frac{n_{f}(\tau)}{\tau} d \tau \leqslant \sigma e t+H(0) \leqslant \sigma_{1} t, \quad t \geqslant 1
$$

где $\sigma_{1}>\sigma$. По теореме о среднем имеем

$$
\left|\frac{1}{\lambda_{k}^{\prime}}-\frac{1}{\lambda_{k}^{\prime \prime}}\right| \leqslant \max _{z} \frac{1}{\left|z^{2}\right|}\left|\lambda_{k}^{\prime}-\lambda_{k}^{\prime \prime}\right| \leqslant \mathrm{const} \cdot \frac{1}{\left|\lambda_{k}^{\prime}\right|^{2}} a\left(\operatorname{Re} \lambda_{k}^{\prime}\right) .
$$

Следовательно, учитывая соотношение (15), получим, что выполняется условие

$$
\begin{aligned}
\sum_{k}\left|\frac{1}{\lambda_{k}^{\prime}}-\frac{1}{\lambda_{k}^{\prime \prime}}\right| & \leqslant \text { const } \cdot \int_{1}^{\infty} \frac{a(t)}{t^{2}} d n(t) \leqslant \text { const } \cdot \int_{1}^{\infty} \frac{1}{t^{2-\varepsilon}} d n(t) \\
& =\text { const } \cdot \int_{1}^{\infty} \frac{n(t)}{t^{3-\varepsilon}} d t \leqslant \text { const } \cdot \int_{1}^{\infty} \frac{1}{t^{2-\varepsilon}} d t<\infty
\end{aligned}
$$

Произведения

$$
v_{1}(\lambda)=\prod_{k}\left(1-\frac{\lambda}{\lambda_{k}^{\prime}}\right) \exp \frac{\lambda}{\lambda_{k}^{\prime}}, \quad \widetilde{v}_{2}(\lambda)=\prod_{k}\left(1-\frac{\lambda}{\lambda_{k}^{\prime \prime}}\right) \exp \frac{\lambda}{\lambda_{k}^{\prime \prime}}
$$

являются целыми функциями [10]. В силу сходимости ряда

$$
\sum_{k}\left|\frac{1}{\lambda_{k}^{\prime}}-\frac{1}{\lambda_{k}^{\prime \prime}}\right|
$$

произведение

$$
v_{2}(\lambda)=\prod_{k}\left(1-\frac{\lambda}{\lambda_{k}^{\prime \prime}}\right) \exp \frac{\lambda}{\lambda_{k}^{\prime}}
$$


также является целой функцией. Тогда функция $f(\lambda) / v_{1}(\lambda) v_{2}(\lambda)$ - это целая функция без нулей, т.е. эта функция имеет вид $\exp g(\lambda)$, где $g(\lambda)$ - целая функция. Положим

$$
f_{i}(\lambda)=v_{i}(\lambda) \exp \frac{g(\lambda)}{2}, \quad i=1,2 .
$$

Функции $f_{i}(\lambda)$ удовлетворяют условиям теоремы 5 . Действительно, по построению $f_{1} f_{2}=f$ и

$$
\ln \left|f_{1}(\lambda)\right|-\ln \left|f_{2}(\lambda)\right|=\sum_{k}\left(\ln \left|1-\frac{\lambda}{\lambda_{k}^{\prime}}\right|-\ln \left|1-\frac{\lambda}{\lambda_{k}^{\prime \prime}}\right|\right) .
$$

Поэтому первое неравенство в теореме 5 сводится к утверждению теоремы 4 . Если $\lambda=r \exp (i \varphi)$ и $r$ достаточно большое, то

$$
\left|\frac{\lambda}{\zeta-\lambda}\right| \leqslant C(\varphi), \quad \zeta \in G_{a} .
$$

По теореме о среднем и из последнего неравенства вытекает оценка

$$
|\ln | 1-\frac{\lambda}{\lambda_{k}^{\prime}}|-\ln | 1-\frac{\lambda}{\lambda_{k}^{\prime \prime}}||=\left|\frac{\left(\lambda_{k}^{\prime}-\lambda_{k}^{\prime \prime}\right) \lambda}{\left(\lambda-\lambda_{k}^{*}\right) \lambda_{k}^{*}}\right| \leqslant C_{1}(\varphi) \frac{a\left(\lambda_{k}^{*}\right)}{\left|\lambda_{k}^{*}\right|},
$$

где $\lambda_{k}^{*}$ - точка, лежащая на отрезке между $\lambda_{k}^{\prime}$ и $\lambda_{k}^{\prime \prime}$. Если $n^{*}(t)$ - число точек $\lambda_{k}^{*}$, лежаших в круге радиуса $t$ с центром в нуле, то из (15) следует, что для $n^{*}(t)$ верна аналогичная оценка:

$$
n^{*}(t) \leqslant \sigma^{*} t, \quad t \geqslant 1 .
$$

Учитывая, кроме того, что для любого $\varepsilon>0 a(t) \leqslant C(\varepsilon) t^{\varepsilon}, t \geqslant 1$, получим оценку

$$
\begin{aligned}
\sum_{\left|\lambda_{k}^{*}\right| \leqslant 2|\lambda|}|\ln | 1-\frac{\lambda}{\lambda_{k}^{\prime}}|-\ln | 1-\frac{\lambda}{\lambda_{k}^{\prime \prime}}|| & \leqslant C_{2}(\varphi, \varepsilon) \sum_{\left|\lambda_{k}^{*}\right| \leqslant 2|\lambda|} \frac{1}{\left|\lambda_{k}^{*}\right|^{1-\varepsilon}} \\
& =C(\varphi, \varepsilon) \int_{1}^{2|\lambda|} \frac{d n^{*}(t)}{t^{1-\varepsilon}} \leqslant C(\varphi, \varepsilon)|\lambda|^{\varepsilon} .
\end{aligned}
$$

Если же $\left|\lambda_{k}^{*}\right|>2|\lambda|$, то $\left|\lambda_{k}^{*}-\lambda\right| \geqslant\left|\lambda_{k}^{*}\right| / 2$, поэтому

$$
\begin{aligned}
\sum_{\left|\lambda_{k}^{*}\right|>2|\lambda|}|\ln | 1-\frac{\lambda}{\lambda_{k}^{\prime}}|-\ln | 1-\frac{\lambda}{\lambda_{k}^{\prime \prime}}|| & \leqslant 2|\lambda| C \sum_{\left|\lambda_{k}^{*}\right|>2|\lambda|} \frac{a\left(\left|\lambda_{k}^{*}\right|\right)}{\left|\lambda_{k}^{*}\right|^{1-\varepsilon}} \\
& =2 C_{\varepsilon}|\lambda| \int_{2|\lambda|}^{\infty} \frac{d n^{*}(t)}{t^{2-\varepsilon}} \leqslant C_{(\varphi, \varepsilon)|\lambda|^{\varepsilon} .}
\end{aligned}
$$

Это неравенство вместе с соотношением (16) дает вторую оценку в утверждении теоремы 5 .

2. Здесь рассмотрим вопрос о факторизации целых функций с множеством нулей типа $\Lambda_{1}$ и $\Lambda_{2}$, тем самым закончим доказательство основной теоремы. 
ТЕОРема 6. Пусть $f$ - целая функция, и нули функции $f$ находятся в полосе $|\operatorname{Im} z| \leqslant 1$, причем в каждом квадрате

$$
K_{n}=\{z: 2 n \leqslant \operatorname{Re} z \leqslant 2(n+1),|\operatorname{Im} z| \leqslant 1\}
$$

находится не более чем один нуль. Тогда функция $f$ представляется в виде произведения цельх функций $f_{1}, f_{2}$ так, что выполняется соотношение

$$
|\ln | f_{1}(\lambda)|-\ln | f_{2}(\lambda)|| \leqslant \text { const } \cdot\left(\ln (|\lambda|+1)+\ln ^{+}(1 / \rho(\lambda))\right),
$$

әде $\rho(\lambda)$ - расстояние от точки $\lambda$ до множества нулей функции $f$.

ДокАЗАТЕЛЬСтво. Пусть $z_{k}, k \in \mathbb{Z},-$ нули функции $f$, перенумерованные в порядке возрастания $\operatorname{Re} z_{k}$. Не уменьшая обшности можем считать, что $\left|\operatorname{Re} z_{k}\right| \geqslant 1$ и $\operatorname{Re} z_{0}=\min \left\{\operatorname{Re} z_{k}: \operatorname{Re} z_{k} \geqslant 0\right\}$. Если положим

$$
\mu_{2 k}=z_{4 k}, \quad \mu_{2 k+1}=z_{4 k+1}, \quad \nu_{2 k}=z_{4 k+2}, \quad \nu_{2 k+1}=z_{4 k+3},
$$

то в силу условия на расположение нулей для любого $k$ имеем $\left|\operatorname{Re} \mu_{k}-\operatorname{Re} \nu_{k}\right| \geqslant 2$. Пусть $\min _{k}\left|\nu_{k}-\lambda\right|=\left|\nu_{k_{0}}-\lambda\right|$. Для $k=k_{0}, k_{0} \pm 1$, воспользуемся оценкой

$$
\begin{aligned}
& |\ln | 1-\frac{\lambda}{\mu_{k}}|| \leqslant \text { const } \cdot\left(\ln (|\lambda|+1)+\ln ^{+}(1 / \rho(\lambda))\right), \\
& |\ln | 1-\frac{\lambda}{\nu_{k}}|| \leqslant \text { const } \cdot\left(\ln (|\lambda|+1)+\ln ^{+}(1 / \rho(\lambda))\right),
\end{aligned}
$$

где $\rho(\lambda)$ - расстояние от точки $\lambda$ до нулей функции $f$, из которой следует соотношение

$$
\sum_{\left|k-k_{0}\right| \leqslant 1}|\ln | 1-\frac{\lambda}{\mu_{k}}|-\ln | 1-\frac{\lambda}{\nu_{k}}|| \leqslant C\left(\ln (|\lambda|+1)+\ln ^{+}(1 / \rho(\lambda))\right) .
$$

Для остальных $k$ используем формулу

$$
\ln \left|1-\frac{\lambda}{\mu_{k}}\right|-\ln \left|1-\frac{\lambda}{\nu_{k}}\right|=\operatorname{Re} \int_{\nu_{k}}^{\mu_{k}} \frac{\lambda}{z(z-\lambda)} d z .
$$

Поскольку $\left|\operatorname{Re} \mu_{k}-\operatorname{Re} \nu_{k}\right| \geqslant 2$ и $\left|\operatorname{Im} \mu_{k}\right|,\left|\operatorname{Im} \nu_{k}\right| \leqslant 1$, то из последней формулы вытекает оценка

$$
|\ln | 1-\frac{\lambda}{\mu_{k}}|-\ln | 1-\frac{\lambda}{\nu_{k}}|| \leqslant A \int_{\operatorname{Re} \nu_{k}}^{\operatorname{Re} \mu_{k}} \frac{|\lambda|}{|t(t-\lambda)|} d t,
$$

где $A$ - некоторая абсолютная постоянная. По построению $\left|\operatorname{Re} \mu_{k}\right|,\left|\operatorname{Re} \nu_{k}\right| \geqslant 1$ и интервалы $\left[\operatorname{Re} \mu_{k}, \operatorname{Re} \nu_{k}\right]$ каждую точку вешественной оси покрывают не более чем два раза. Следовательно,

$$
\begin{aligned}
\sum_{\left|k-k_{0}\right|>1}|\ln | 1-\frac{\lambda}{\mu_{k}}|-\ln | 1-\frac{\lambda}{\nu_{k}}|| \\
\leqslant 2 A \int_{|t|>1,|t-\lambda|>1} \frac{|\lambda|}{|t(t-\lambda)|} d t \leqslant A_{1} \ln (|\lambda|+1), \quad \lambda \in \mathbb{C} .
\end{aligned}
$$


Вместе с соотношением (17) эта оценка дает неравенство

$$
\sum_{k=-\infty}^{\infty}|\ln | 1-\frac{\lambda}{\mu_{k}}|-\ln | 1-\frac{\lambda}{\nu_{k}}|| \leqslant C\left(\ln (|\lambda|+1)+\ln ^{+}(1 / \rho(\lambda))\right)
$$

Рассмотрим целые функции

$$
\widetilde{v}_{1}(\lambda)=\prod_{k}\left(1-\frac{\lambda}{\mu_{k}}\right) \exp \frac{\lambda}{\mu_{k}}, \quad \widetilde{v}_{2}(\lambda)=\prod_{k}\left(1-\frac{\lambda}{\nu_{k}}\right) \exp \frac{\lambda}{\nu_{k}} .
$$

Сходимость произведения в определении функции $\widetilde{v}_{1}$ очевидна. Сходимость произведения для $\widetilde{v}_{2}$ следует из соотношения (18). По построению $\widetilde{v}_{1}(\lambda) \widetilde{v}_{1}(\lambda)=$ $f(\lambda) \exp g(\lambda)$, где $g$ - целая функция. Положим

$$
f_{j}(\lambda)=\widetilde{v}_{j}(\lambda) \exp \frac{g(\lambda)}{2}
$$

Ясно, что функции $f_{j}$ удовлетворяют условиям теоремы 6.

ТЕОРЕМА 7. Пусть $f$ - целая функция, все нули ее лехсат в квадратах $\pm Q_{n}, \pm Q_{m}^{\prime}$, и в каждом квадрате лежит не более одного нуля. Тогда функиия $f$ представляется в виде произведения цельх функиий $f_{1}, f_{2}$, причем выполняется соотношение

$$
|\ln | f_{1}(\lambda)|-\ln | f_{2}(\lambda)|| \leqslant \operatorname{const}\left(\ln (|\lambda|+1)+\ln ^{+}(1 / \rho(\lambda))\right),
$$

где $\rho(\lambda)$ - расстояние от точки $\lambda$ до нулей функиии $f$.

Эта теорема доказывается точно так же, как и теорема 6. Из утверждений теорем 1, 4, 6, 7 следует доказательство основной теоремы.

TЕОРема 8. Пусть $\varphi \in \mathscr{D}(\mathbb{R})$ и нули функиии $\widehat{\varphi}(\lambda)$ лежат в области

$$
G=\{z=x+i y:|y| \leqslant \exp a(\sqrt{\ln (|x|+1)})\}
$$

Если носитель функиии ч лежст в интервале $[-2 N, 2 N]$, то $\varphi$ может бъть представлена как свертка функиий $\varphi_{1}, \varphi_{2} \in \mathscr{D}(\mathbb{R})$, причем носители функиий $\varphi_{j}$ лехсат в интервале $[-N, N]$.

ДокАЗАТЕЛЬСтво. Пусть $\widehat{\varphi}(\lambda)=\lambda^{k} f(\lambda), f(0) \neq 0 . \mathrm{K}$ функции $f(\lambda)$ применим основную теорему, полагая

$$
F(\lambda)=\frac{f(\lambda)}{f(0)}
$$

$$
H(\lambda)=2 N|\operatorname{Im} \lambda|+M
$$


для подходящей постоянной $M$. Тогда функция $\widehat{\varphi}$ представляется в виде произведения целых функций $f_{1}, f_{2}$ так, что

$$
\begin{aligned}
|\ln | f_{1}(x)|-\ln | f_{2}(x)|| \leqslant \frac{C}{p}(M- & \ln |f(x)|+\ln |f(0)|) \\
& +C_{1} \ln (|x|+1)+C_{2} \ln ^{+}(1 / \rho(\lambda))+C_{3} e^{p} .
\end{aligned}
$$

Тогда выполняется оценка

$$
\begin{aligned}
\ln \left|f_{j}(x)\right| \leqslant\left(\frac{1}{2}-\frac{C}{2 p}\right) \ln |f(x)|+ & \frac{M C+\ln |f(0)|}{2 p} \\
& +C_{1} \ln (|x|+1)+C_{2} \ln ^{+}(1 / \rho(x))+C_{3}^{\prime} e^{p} .
\end{aligned}
$$

Выбирая величину $p \geqslant 2 C$ получим, что для всех $x, \rho(x) \geqslant(|x|+1)^{-3}$, вьполняется оценка

$$
\ln \left|f_{j}(x)\right| \leqslant \frac{1}{4} \ln |f(x)|+\text { const } \cdot \ln (|x|+1) \leqslant \text { const } \cdot \ln (|x|+1) .
$$

Исключительное множество $E=\left\{x: \rho(x) \leqslant(|x|+1)^{-3}\right\}$ может быть покрыто системой кругов с конечной суммой радиусов. Этот факт доказывается стандартными методами теории субгармонических функций на основе леммы Хеймана (см. $[11$, с. 246$])$. В результате мы получим, что функции $f_{j}$ лежат в $\widehat{\mathscr{D}}(\mathbb{R})$, т.е. являются преобразованием Фурье-Лапласа функций $\varphi_{j} \in \mathscr{D}(\mathbb{R})$. Что касается носителей функций $\varphi_{j}$, то нужно воспользоваться последней оценкой в утверждении основной теоремы.

ТЕОрема 9. Пусть $\mu$-распределение конечного порядка с компактным носителем в интервале $[-2 N, 2 N]$. Если нули функиии

$$
\widehat{\mu}(\lambda)=\mu(\exp (i \lambda t))
$$

лежсат в области $G$ (см. формулировку теоремы 8), то распределение $\mu$ представляется в виде свертки двух распределений $\mu_{1}, \mu_{2}$ с носителями в интервале $[-N, N]$.

ДокАЗАТЕЛЬСтво. Преобразование Фурье-Лапласа распределения конечного порядка с компактньм носителем - это целая функция экспоненциального типа, удовлетворяюшая условию на вешественной оси

$$
\ln |f(x)| \leqslant C \ln (|x|+1)+M \text {. }
$$

Поэтому теорема 9 может быть доказана так же, как и теорема 8 , если в качестве функции $H(\lambda)$ взять функцию $2 N|\operatorname{Im} \lambda|+C \ln (|\lambda|+1)+M$, с подходящими постоянныги $C$ и $M$. 


\section{Список литературы}

1. Ehrenpreis L. Solution of some problems of division. IV // Amer. J. Math. 1960. V. 82. P. 522-588.

2. Хермандер Л. Анализ линейных дифференциалњыт операторов с частньми производными 2. Дифференциальные операторы с постоянными коэффициентами. М.: Мир, 1986.

3. Demaily J. P. Construction d'hipersurfaces irreductibles avec lien singulier donne dans $\mathbb{C}^{n}$ // Ann. Inst. Fourier. 1980. V. 30. № 3. P. 219-236.

4. Malliaven P., Dixmier J. Factorisations de fonctions et de vecteurs indefeniment differentiables // Bull. Sci. Math. 1978. V. 102. № 2. P. 305-330.

5. Rubel L. A., Aquires W.A., Taylor B. A. Irreducibility of certain entier functions and applications to harmonic analysis // Ann. of Math. 1978. V. 108. № 2. P. 553-567.

6. Dickson D. G. Factoring rapidly decreasing entire function // Bull. Sci. Math. 1986. V. 110. № 4. P. 335-345.

7. Dickson D. G. Factoring functions of exponential type with restriction on a line // Bull. Sci. Math. 1988. V. 112. № 2. P. 77-100.

8. Юлмухаметов Р. С. Аппроксимация субгармонических функций // Anal. Math. 1985. T. 11. C. $257-282$.

9. Юлмухаметов Р. С. Расщеплениецелых функций с нулями в полосе // Матем. сб. 1995. T. 186. № 7. С. 147-160.

10. Левин Б. Я. Распределение корней целых функций. М.: Гостехиздат, 1956.

11. Ландкоф Н. С. Основы современной теории потенциала. М.: Наука, 1966. 